\title{
CANPROFESSIONAL QUALIFICATION CHANGE PHYSICAL THERAPISTS' PERCEPTION ABOUT KNEE OSTEOARTHRITIS TREATMENT? RESULTS OF A CROSS-SECTIONAL SURVEY IN THE AMAZON REGION
}

Natália Camargo Rodrigues losimuta ${ }^{1}$, Nívea Renata Oliveira Monteiro² , Débora Juliana do Rosário ${ }^{1}$, Vânia Tiê Koga Ferreira ${ }^{1}$, Daniela Gonçalves Ohara ${ }^{1}$, Maycon Sousa Pegorari1, Areolino Pena Matos ${ }^{1, \star}$

1.Universidade Federal do Amapá, Macapá (AP), Brazil. 2.Universidade de São Paulo, Ribeirão Preto (SP), Brazil.

*Corresponding author: areolino.matos@gmail.com

\section{BACKGROUND}

Osteoarthritis $(\mathrm{OA})$ is the most prevalent form of arthritis, which requires appropriate therapeutic intervention ${ }^{1}$. Physical therapy plays a central role in its management ${ }^{2}$. However, little is known about the beliefs and interventions of physical therapists who treat OA in Brazil. The aim of this study is to determinate whether there is association between the professional qualification of physical therapists (PTs) in the Amazon region of Brazil and their perception of efficiency or not of therapeutic interventions for knee osteoarthritis (OA).

\section{MATERIALS AND METHODS}

Cross-sectional online survey study. The professional's efficiency perception was assessed using a list of 20 interventions for a hypothetical case of OA. Data were analyzed with descriptive and inferential analysis using the chi-square test and binary logistic regression.

\section{RESULTS}

A total of 370 professionals responded. Graduated PTs were more likely to recognize as efficient: ultrasound (odds ratio, OR: 0.17; confidence interval, $\mathrm{Cl}$ : 0.06-0.43), short-wave (OR: 0.45; Cl: 0.23-0.96), transcutaneous electrical nerve stimulation (TENS) and

Table 1. Association between professional qualification and perception of efficiency or inefficiency of interventions with significant differences.

\begin{tabular}{|c|c|c|}
\hline Interventions & Professional qualification & Odds ratio $(95 \% \mathrm{Cl})$ \\
\hline \multirow{2}{*}{ Ultrasound } & Graduation & $0.17(0.06-0.43)^{\star}$ \\
\hline & Specialization/Residency & $0.50(0.25-0.96)^{*}$ \\
\hline \multirow{2}{*}{ Short-wave diathermy } & Graduation & $0.45(0.23-0.89)^{\star}$ \\
\hline & Specialization/Residency & $0.83(0.46-1.52)$ \\
\hline \multirow{2}{*}{ TENS and EE } & Graduation & $0.26(0.11-0.62)^{\star}$ \\
\hline & Specialization/Residency & $0.43(0.22-0.86)^{\star}$ \\
\hline \multirow{2}{*}{ Cryotherapy } & Graduation & $0.42(0.21-0.84)^{*}$ \\
\hline & Specialization/Residency & $0.88(0.48-1.61)$ \\
\hline \multirow{2}{*}{ Heat } & Graduation & $0.46(0.23-0.91)^{\star}$ \\
\hline & Specialization/Residency & $0.85(0.46-1.55)$ \\
\hline \multirow{2}{*}{ Whirpool therapy } & Graduation & $0.28(0.14-0.57)^{\star}$ \\
\hline & Specialization/Residency & $0.72(0.39-1.35)$ \\
\hline \multirow{2}{*}{ Muscle strengthening } & Graduation & $0.09(0.01-0.86)^{\star}$ \\
\hline & Specialization/Residency & $0.28(0.08-0.96)^{\star}$ \\
\hline \multirow{2}{*}{ Kinesiology tape } & Graduation & $0.41(0.20-0.81)^{\star}$ \\
\hline & Specialization/Residency & $0.50(0.27-0.92)^{\star}$ \\
\hline \multirow{2}{*}{ Rest } & Graduation & $0.40(0.20-0.78)^{\star}$ \\
\hline & Specialization/Residency & $0.56(0.30-1.03)$ \\
\hline
\end{tabular}

${ }^{*} \mathrm{p}<0.05$, according to binary logistic regression analysis; TENS: transcutaneous electrical nerve stimulation; EE: electrical stimulation. 
electrical stimulation (EE) (OR: 0.26; Cl: 0.11-0.62), cryotherapy (OR: 0.42; Cl: 0.21-0.84), heat (OR: 0.43; Cl: 0.23-0.91), whirlpool (OR: 0.28; Cl: 0.14-0.57), muscle strengthening (OR: 0.09; Cl: 0.01-0.86), kinesiology tape (OR: 0.41; Cl: 0.20-0.81) and rest (OR: 0.40; Cl: 0.20-0.78). The same association occurred for ultrasound (OR: 0.50; Cl: 0.25-0.96), TENS (OR: 0.43; Cl: 0.22-0.86), muscle strengthening (OR: 0.28; $\mathrm{Cl}$ : 0.08-0.96) and kinesiology tape (OR: 0.50; $\mathrm{Cl}$ : 0.27-0.92) among specialists (Table 1).

\section{CONCLUSION}

There was an inverse association between professional qualification and the perception of interventions efficiency. The lower academic degree leads a lower chance to consider poorly efficient or inefficient some not recommended interventions for knee OA.

\section{FUNDING}

This study received funding by Programa de Auxílio a Pesquisador from Universidade Federal do Amapá (PAPESQ/UNIFAP) 2019.

\section{REFERENCES}

1. Hunter DJ; Bierma-Zeinstra S. Osteoarthritis. Lancet. 2019;393(10182):1745-59. https://doi.org/10.1016/S0140-6736(19)30417-9

2. Collins NJ, Hart, HF, Mills KAG. Osteoarthritis year in review 2018: rehabilitation and outcomes. Osteoarthr Cartil . 2019;27(3):378-91. https:// doi.org/10.1016/j.joca.2018.11.010 